\title{
RFID Based Security and Access Control System
}

\author{
Umar Farooq, Mahmood ul Hasan, Muhammad Amar, Athar Hanif, and Muhammad Usman Asad
}

\begin{abstract}
This paper describes the design of RFID based security and access control system for use in hostels inside the Punjab University premises. The system combines RFID technology and biometrics to accomplish the required task. When the RFID reader installed at the entrance of hostel detects a number, the system captures the user image and scans the database for a match. If both the card and captured image belong to a registered user, access is granted; otherwise the system turns on the alarm and makes an emergency call to the security van through GSM modem. In this way, the suspicious persons can be caught.
\end{abstract}

Index Terms-Security and access control, RFID, face recognition.

\section{INTRODUCTION}

Automatic identification and access control system has become necessary to overcome the security threats faced by many organizations in Pakistan these days. By installing the system at the entrance will only allow the authorized persons to enter the organization. The system can also be installed at various points inside the organization to track the person's movement and to restrict their access to sensitive areas in the organization. In such a way, suspicious persons can be caught which will surely improve the security level in the organization.

Radio frequency identification (RFID) is a wireless technology that can be used to develop the access control system. The literature has revealed the use of this technology to automate various processes ranging from industrial sector to home control [1]. Bo Yan [2] has reported the use of RFID technology to automate sight spot ticket management system. The system hardware consists of RFID electronic tickets, RFID readers, computer terminals, optical networks, computer servers and site controllers. Electronic ticket contains the S-DES encrypted form of data including scenic region number, scenic spot number, ticket type, ticket date, site number, serial number and check bit. The RFID reader at the site reads the data inside the e-ticket and transmits it to the computer terminal and servers though the network. The data is decrypted at the terminal and its authenticity is verified. The site controller then allows the right tourist to enter the spot. This system identification and authentication process is carried out at three sub-levels namely the sale sub- system, the

Manuscript received May 27, 2013; revised August 22, 2013.

Umar Farooq and Muhammad Amar are with Department of Electrical Engineering, University of The Punjab Lahore (e-mail: engr.umarfarooq@yahoo.com; amar.ete611@yahoo.com).

Mahmood ul Hasan is with Department of Electrical Engineering, Mirpur University of Science \& Technology (e-mail: mahmood.uet@gmail.com).

Athar Hanif and Muhammad Usman Asad are with Department of Electrical Engineering, The University of Lahore (e-mail: athar.hanif@ee.uol..edu.pk, usmanasad01@ @otmail.com). decision sub-system and the management sub-system. All these processes communicate with each other through database information. G. Ostojic [3] has developed an automatic vehicle parking control system based on RFID technology in the city of Novi Sad, Republic of Serbia. The hardware of the system consists of RFID tag and reader operating at a frequency of $13.56 \mathrm{MHz}$ for authentication, inductive loop for metal detection, a capacity sensor for counting vehicles, Siemens MC 39i GPRS modem for communication between entrance and exit gates and FEC FC440 programmable logic controller (PLC) which is the heart of the system. When the car stops on the inductive loop at the entrance, RFID tag is read by the reader. The data on the tag includes the unique identification number (UID), validity period and check bit for checking the parking status. This data is manipulated by PLC and access is granted for parking the vehicle if tagged information contains correct UID, validity period and parking status. After the vehicle has entered the parking lot, its parking status will be changed by the RFID reader/writer to prevent the entry of another vehicle on the same card. The same procedure is repeated when the vehicle is leaving the parking lot. Nova Ahmed [4] has described RFID based indoor guidance and monitoring system known as GuardianAngel in pervasive environment. The beauty of the system is that it can generate dynamic queries in real time through user interface. The environment in the system is equipped with RFID tags and is divided into various zones. The middleware of the system is divided into two layers namely guidance layer and monitoring layer. The guidance layer is provided with handheld RFID reader to provide locality information to the monitoring layer periodically. Thus monitoring layer has the information of the entire environment. Experimental results have shown that system is nearly $100 \%$ accurate in providing the zonal information thereby allowing the construction of very robust guidance and monitoring applications. Kuo-shien Huang [5] has described a business model based approach for utilizing RFID technology in automating the process according to enterprise strategic vision and goals. The author has built a business model for bike renting system and used RFID technology to implement the system. The conventional way of getting the bike on rent which includes the recording of customer data by pen and then inputting the data to the computer is replaced by providing a RFID card to the customer and fixing an RFID tag on the bike. The bike is tagged to keep track of its location from the renting store to the return store. The information is shared amongst the stores through web interface. In this way, successful RFID strategy is built and deployed.

This paper discusses the design of a security and access control system using RFID technology. The system is comprised of three modules namely entrance monitoring, exit monitoring and mess monitoring. These modules 
communicate to the computer system through main controller. After the information from these modules is processed by the computer, the control commands are issued to the modules for granting or denying access to the user.

TABLE I: COMPARISON OF ACTIVE \& PASSIVE RFID TAGS

\begin{tabular}{|c|c|c|}
\hline Attribute & Active Tags & Passive Tags \\
\hline Source of Power & Built-in Battery & Electromagnetic Induction \\
\hline Reading Distance & High (20 to $100 \mathrm{~m})$ & Low (Up to $3 \mathrm{~m}$ ) \\
\hline $\begin{array}{l}\text { Required Signal } \\
\text { Strength }\end{array}$ & Low & High \\
\hline Tag Cost & High $(\$ 15$ to $\$ 100)$ & Low $(\$ 0.15$ to $\$ 5)$ \\
\hline Data Storage & $\begin{array}{l}\text { Large read/write data } \\
\qquad(128 \mathrm{~kb})\end{array}$ & $\begin{array}{c}\text { Small read/write } \\
(128 \mathrm{~b})\end{array}$ \\
\hline Size & Large & Small \\
\hline Weight & Large & Small \\
\hline Tag Life & $\begin{array}{l}\text { Small ( } 3 \text { to } 8 \text { years } \\
\text { depending upon tag } \\
\text { broadcast rate) }\end{array}$ & $\begin{array}{l}\text { Large (Up to } 10 \text { years } \\
\text { depending upon the } \\
\text { environment the tag is in) }\end{array}$ \\
\hline Tag Readers & Typically lower in cost & Typically higher in cost \\
\hline $\begin{array}{l}\text { Industries/ } \\
\text { Applications }\end{array}$ & $\begin{array}{c}\text { Auto dealership } \\
\text { Auto Manufacturing } \\
\text { Hospitals } \\
\text { Construction } \\
\text { Mining } \\
\text { Laboratories } \\
\text { Remote monitoring }\end{array}$ & $\begin{array}{c}\text { Supply Chain } \\
\text { High vol. Manufacturing } \\
\text { Libraries/book stores } \\
\text { Pharmaceuticals } \\
\text { Passport } \\
\text { Electronic tolls } \\
\text { Item level tracking }\end{array}$ \\
\hline
\end{tabular}

\section{OVERVIEW OF RFID TECHNOLOGY}

RFID system consists of three components namely transponder (tag), interrogator (reader) and computer containing the database, as shown in Fig. 1. The interrogator reads the tag data and transmits it to the computer for authentication. The information is processed and upon verification, access is granted. The system offers diverse frequency band ranging from low frequencies to microwave frequencies [5]:

- Low Frequency: 125-134 KHz

- High Frequency: $13.56 \mathrm{MHz}$

- Ultra High Frequency: 902-928 MHz

- Microwave Frequency: $2.4 \mathrm{GHz}$

Depending upon the source of electrical energy, RFID tags are classified as either active or passive. The active tags use a battery for powering the circuit on the tag and transmit the tag information upon the reader request. However, these tags are very expensive and seldom used. On the other hands, passive tags get energy from the reader to power their circuit. These tags are very cost-effective and hence most of the applications use them. A comparison of these tags highlighting important features is shown in Table I [6]-[8].

In the present work, passive RFID tags have been used. A passive RFID tag transmits information to the reader when it comes in the vicinity of electromagnetic field generated by the reader. The phenomenon is based on Faraday's law of electromagnetic induction. The current flowing through the coil of interrogator produces a magnetic field which links to the transponder coil thereby producing a current in the transponder coil. The transponder coil then varies this current by changing the load on its antenna. This variation is actually the modulated signal (scheme is known as load modulation) which is received by the interrogator coil through mutual induction between the coils. The interrogator coil decodes this signal and passes to the computer for further processing.

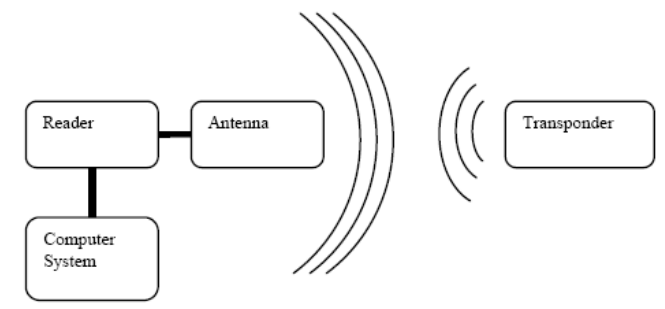

Fig. 1. Basic RFID system.

\section{RELATED WORK}

Many researchers have utilized RFID technology in developing access control system. Filipe [7] has developed an RFID based monitoring and access control system consisting of RFID terminal, camera, server and an alert device. Upon detecting a transponder, the terminal captures a photo and transmits the data including the UID and photo to the server through TCP/IP connection. The server searchers the database for this particular query and sends the results back to the terminal to allow or deny the access. The system also monitors illicit acts e.g., a person tries to enter when the door is open without completion of authentication process and turns on the alert device using web services. The performance of the system is tested by installing RFID kits with antennas covering a range of $10 \mathrm{~cm}$ and satisfactory results are obtained. Xiang-Lei Meng [9] has described an RFID based embedded security authentication system with novel face recognition structure. The system comprises of two phases namely registration and recognition. In registration phase, ten pictures of user face with different emotions are collected and eigen information is obtained with an extraction algorithm. This information along with a UID is written on RFID tag. In recognition phase, a camera tracks the face and an extraction algorithm returns eigen information of the face in the picture. This information is then matched with the information already stored on the tag for authentication. The entire processing is done on embedded ARM11 processor, S3C6410 instead of computer terminal/server which has resulted in faster response time, about $57 \mathrm{~ms}$ with authentication accuracy up to $86.5 \%$. The performance of the system is compared with the existing database systems and is found to have far better response time with the same authentication accuracy. Dong-Liang Wu [10] has described an access control system based on RFID in conjunction with face recognition based on neural network. The system recognizes the face of person holding RFID card and denies the access if person is found to be unauthorized. Radial basis function neural network (RBFNN) has been used for learning the face of authorized persons. Principal component analysis (PCA) has been used for extracting the features from the image and linear discriminant analysis (LDA) for refining these features. The network is trained with localized generalization error model (L-GEM) for enhancing its generalization capabilities. 
Experimental results have shown that proposed system can improve the security of RFID access control.

\section{SYSTEM COMPONENTS}

\section{A. RFID Tag}

IPC80 passive RFID tag operating at a frequency of $125 \mathrm{KHz}$ is issued to the user. The tag transmits information to the reader in ASK format [11].

\section{B. RFID Reader}

IP10 proximity card reader with operating frequency of $125 \mathrm{KHz}$ and reading distance up to 4 inches is used. The reader can be easily installed on metal doors, provides the tag information serially in RS232 format and is suitable for indoor as well as outdoor operations [11]. Three such readers are installed for hostel security: hostel entrance gate, hostel exit gate and mess entrance gate.

\section{Camera}

Logitech C500 webcam is used to capture images. The camera has 1.3 mega pixel sensors and can capture video up to $1280 \times 1024$ pixels. Two such cameras are installed for hostel security: one at the entrance and other at the exit. No camera is used for entering in the mess hall to reduce the overall complexity.

\section{GSM Modem}

Nokia $12 \mathrm{i}$ GSM modem is used to make emergency call to the security van. Nokia $12 \mathrm{i}$ offers advance GSM connectivity and supports EDGE/GPRS and HSCSD with automated GSM connection establishment it is equipped to provide reliable remote connections and offers application level watchdogs, inbuilt self check mechanisms and a reliable Virtual Machine (VM) for JAVA ${ }^{\mathrm{TM}}$. Nokia $12 \mathrm{i}$ also supports reliable inbuilt internet protocols: TCP/IP for reliable data transfer, UDP/IP for audio and video streaming and HTTP for accessing web pages. The module can also be connected to an external GPS device that supports National Marine Electronics Association (NMEA) standard. The inbuilt NMEA parser can parse the location data from the output that it receives from the GPS device. External microcontroller can use AT commands to communicate with Nokia $12 \mathrm{i}$ and simple remote I/O applications can easily be controlled via text messages.

\section{E. Microcontroller}

AT89C52 microcontroller is selected because it is a powerful microcomputer which has low power consumption and provides a highly flexible and cost-effective solution to many embedded control applications. It has $8 \mathrm{~K}$ bytes of in system reprogrammable flash memory, 256 bytes of internal RAM, 32 programmable I/O lines, three 16 bit timers/counters, eight interrupt sources and a programmable serial channel [11].

\section{F. Nonvolatile RAM}

256K Nonvolatile RAM (NV-Ram) DS1230Y-85 is used for storing passwords against registered RFID numbers. NV-RAM is selected because it combines the best of RAM and ROM: the read and write ability of RAM and non-volatility of ROM. The DS1230 Nonvolatile SRAM is 262,144-bit, fully static, nonvolatile SRAM organized as 32,768 words by 8 bits. Each NV SRAM has a self-contained lithium energy source and control circuitry which constantly monitors $\mathrm{V}_{\mathrm{CC}}$ for an out-of-tolerance condition. When such a condition occurs, the lithium energy source is automatically switched on and write protection is unconditionally enabled to prevent data corruption.

\section{G. Door Locks}

Solenoid operated door locks are used in entrance, exit and mess gates of hostels. A relay is used to energize the solenoid to open the gate.

\section{H. Alarms}

Two alarms are installed; one at the entrance and other at the exit gate. These alarms are turned on if an illegal person tries to enter the hostel premises.

\section{SySTEM OPERATION}

The security and access control system is comprised of two phases: registration phase and recognition phase. In the registration phase, ten images of the hostel user are captured while issuing an RFID tag. These images are used to train a feed forward neural network with back propagation training algorithm and the converged weights are stored corresponding to a particular user. The recognition phase comes when the user wants to enter the hostel. At this point, after getting RFID user number, image of the user is captured and passed to the neural network for recognition. If a match is found, access is granted to the user. The user authenticity is checked at three places: hostel entrance, hostel exit and mess entrance. The entrance and exit modules use RFID and face recognition for identification while mess module use RFID with a password to grant permission. These modules communicate with computer system through a main controller. The main controller transmits the modules information to the computer system. The computer system after processing these interrupts issues commands to the modules through main controller. The data exchange between the main controller and computer system is through serial port while parallel port data and control lines are used for handshaking purposes. The block diagram of the system is shown in Fig. 2.

\section{A. Entrance Monitoring Controller}

Entrance monitoring controller comprises of an RFID reader, a GSM modem, a NV RAM, door lock, alarm, scroll keys and 16x4 LCD; all interfaced to AT89C52 microcontroller as shown in Fig. 3. After detecting and receiving RFID tag data through a serial interrupt routine, microcontroller searches the NV-RAM for this number. If no match is found, the microcontroller makes an emergency call to the security van through GSM modem. At the same time, it sends a request to the computer system through main controller to capture the user image and turns on the alarm signal. On the other hand, if a match is found, the microcontroller checks the entrance status of the user by scanning NV-RAM. If the user has not entered in the hostel yet, the controller sends a request to the computer system to 
capture and process the user image. The computer system performs two functions. First, it verifies the user against the received RFID number using face recognition algorithm and then checks whether the user is a defaulter or not. After processing, the computer system generates one of the three messages: 'access granted' corresponding to registered and clear user, 'access denied' corresponding to a non-registered user and 'user is a defaulter' corresponding to a registered and defaulter user. In case of non-registered user, heavy fine is included in user hostel dues on an account of using the RFID tag of other user. The entrance controller receives the message from computer system and displays it on $16 \times 4$ LCD. The details on LCD can be read with the help of scroll keys. If the message 'access granted' is received by the controller, it opens the entrance gate by switching the relay. At the same time, entrance status of the user is updated in the non volatile RAM. The entrance monitoring module thus ensures the entry of registered and clear users in the hostel and also helps in catching suspicious persons that are not authorized to enter. The flow chart describing the operation of entrance monitoring module is shown in Fig. 4.

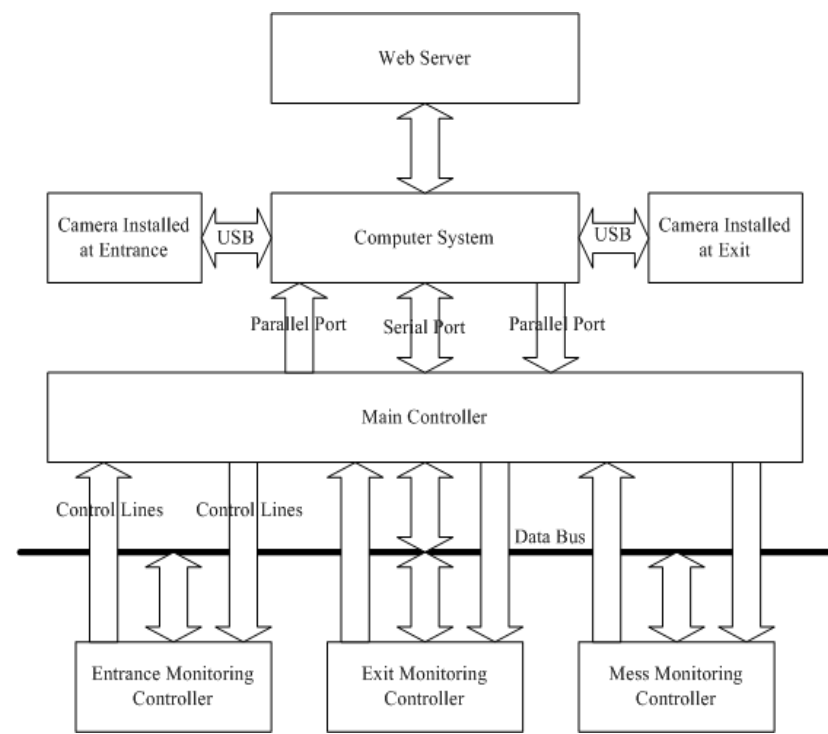

Fig. 2. Block diagram showing modules interconnection with the computer System.

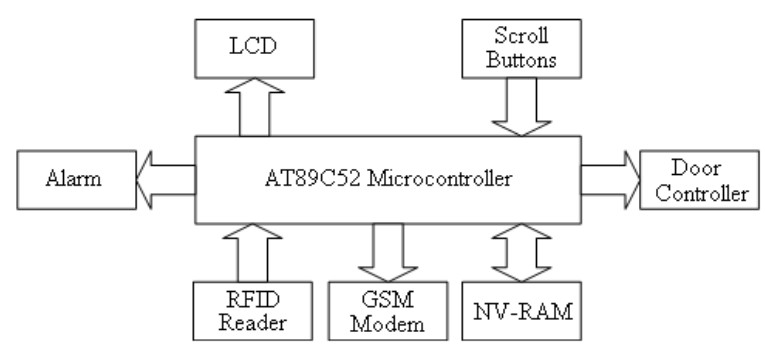

Fig. 3. Block diagram of entrance monitoring controller.

\section{B. Exit Monitoring Controller}

The exit monitoring module comprises of RFID reader, an alarm and door lock; all interfaced to AT89C52 microcontroller as shown in Fig. 5. After receiving RFID tag information through reader, microcontroller sends a request to the entrance monitoring controller to search the user and its entrance status in NV-RAM. Upon receiving a 'no' signal from the entrance controller, exit controller blocks RFID tag and sends a request to the computer system to capture the user image. At the same time, it makes an emergency call through GSM modem interfaced to entrance controller and turns on alarm signal. On the other hand, if a 'yes' signal is received, the exit controller sends a request to computer system to capture and process user image. The computer system uses face recognition algorithm to verify the user. If a match is found, computer system asks the exit controller to grant permission to the user to leave the hostel. After the user has left the hostel, exit controller sends a request to the entrance controller through main controller to update the user entrance status. In case of non-registered user, heavy fine is included in hostel dues of the user for using the card of other person. The exit monitoring controller thus enables the system to allow the valid person to leave the hostel. The flow chart describing the operation of exit monitoring controller is shown in Fig. 6.

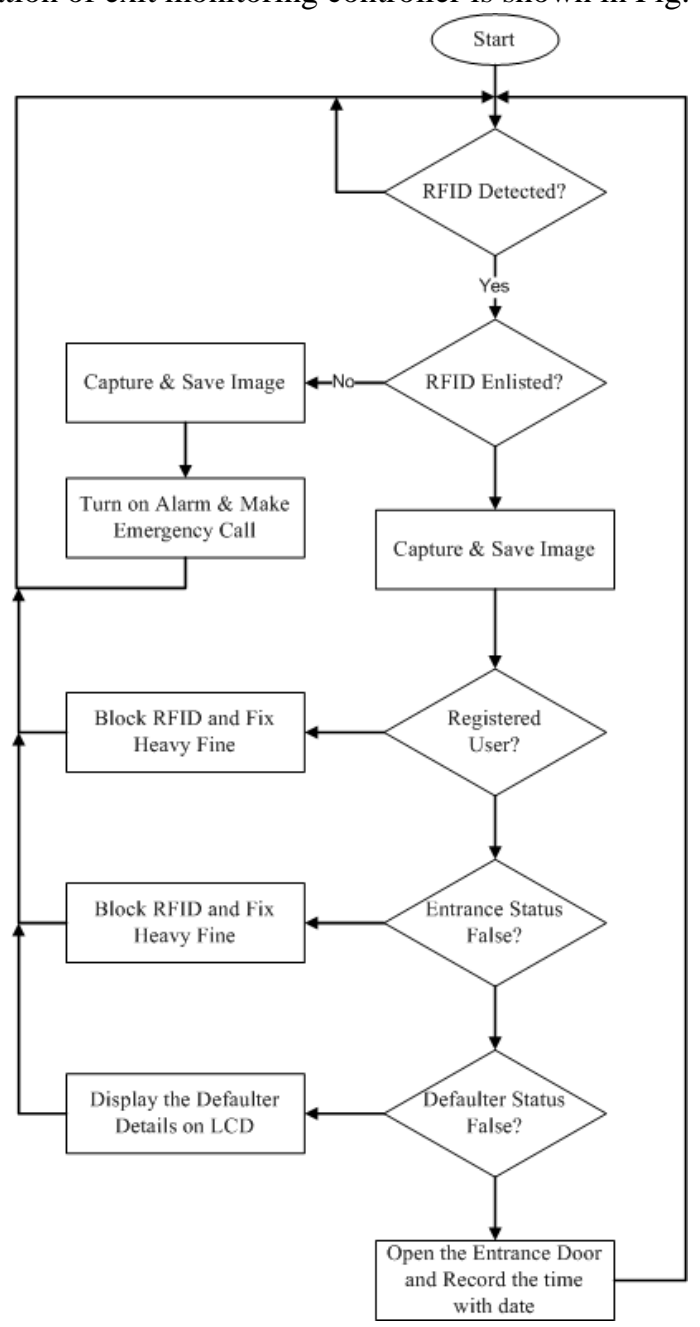

Fig. 4. Flow Chart for entrance monitoring module.

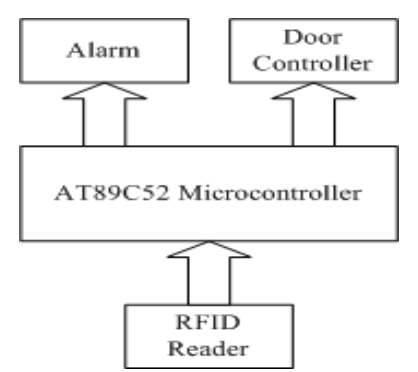

Fig. 5. Block diagram of exit monitoring controller. 


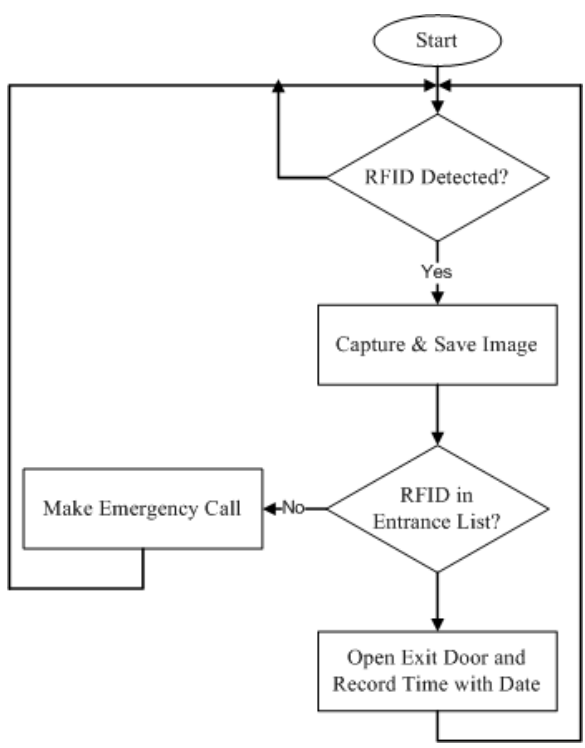

Fig. 6. Flow chart for exit monitoring controller.

\section{Mess Monitoring Controller}

While entrance and exit monitoring controllers help in tracking the users, mess monitoring controller automates the mess attendance system. The mess monitoring module comprises of an RFID reader, 4x3 keypad, 16x4 LCD, a NV-RAM, door lock and alarm indicator; all interfaced to AT89C52 microcontroller as shown in Fig. 7. Upon receiving RFID tag number, the controller searches it in a list of registered numbers stored in NV-RAM. If a match is found, controller asks the user to enter the password. The password list of registered users is also maintained in NV-RAM corresponding to RFID tag numbers. If the entered password is correct, controller grants access to the user for entering the mess hall. At the same time, controller sends the user information along with mess attendance to the computer system through main controller. The computer system updates the database and sends 'attendance recorded' message to the mess controller. In this way, the exact mess charges are maintained in on-line database. The flow chart describing the operation of mess monitoring controller is shown in Fig. 8.

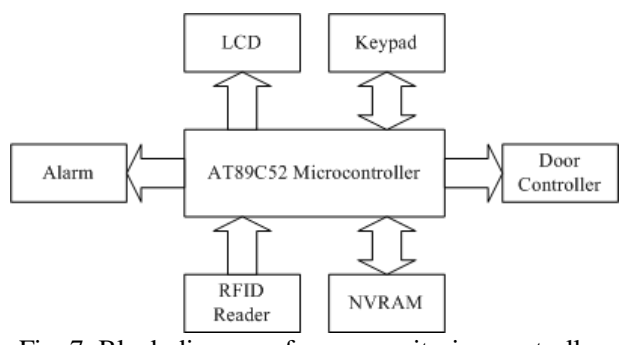

Fig. 7. Block diagram of mess monitoring controller.

\section{Computer System and Web Server}

The information provided by the system modules is maintained in a database inside the computer system. The database keeps record of user history including check-in time and date, check-out time and date, electricity, gas and mess charges, fine (penalty) details, RFID card lost details and user's visitor record. In addition to this, database also keeps record of illegal persons trying to enter the hostel premises. A screen short of a user form in database is shown in Fig. 9. The security and access control system is installed in eighteen hostels and computer systems in these hostels share the information to a web server which is the central station and can be searched to locate a particular user inside the hostels.

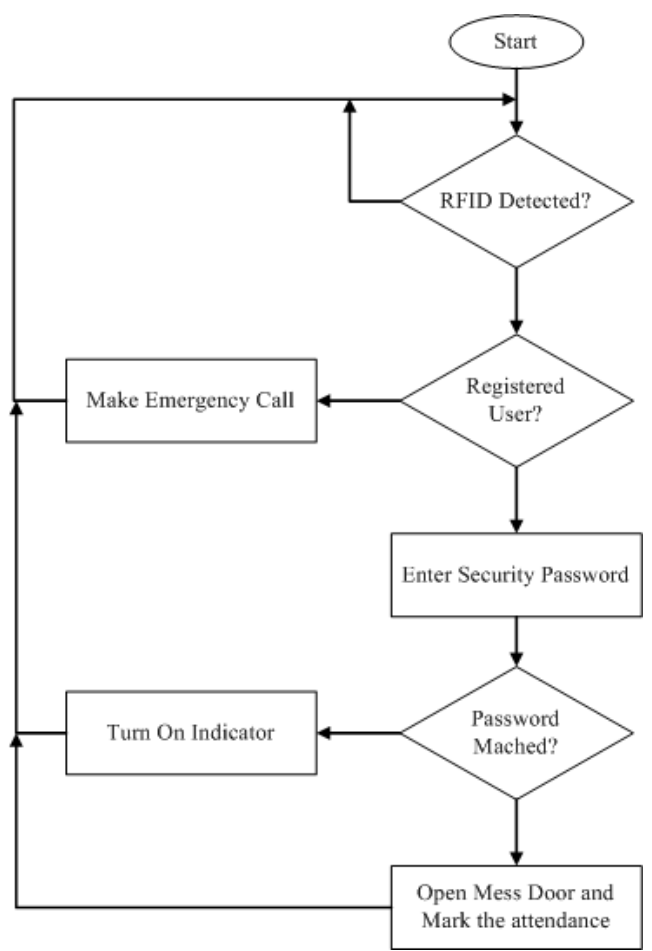

Fig. 8. Flow chart for mess monitoring controller.

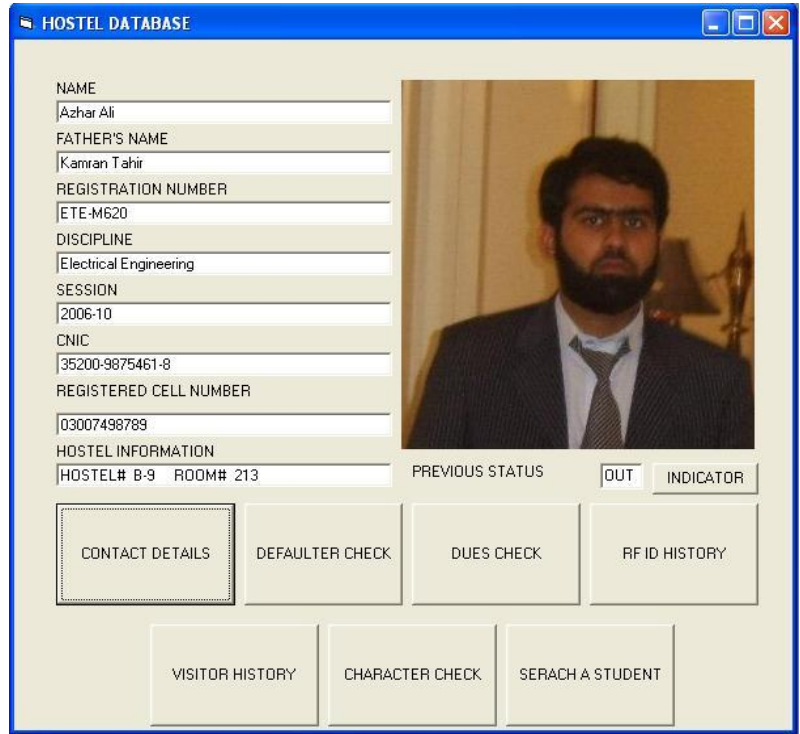

Fig. 9. A screenshot of user form in hostel database.

\section{CONCLUSIONS}

In this paper, design of a security and access control system for use in Punjab University hostels is presented. The system uses radio frequency identification with biometrics technology to differentiate between valid and invalid users. The system accomplishes the security and access control task by processing information from sub-controllers. These controllers include entrance monitoring controller, exit monitoring controller and mess monitoring controller installed at entrance gate, exit gate and mess gate respectively. These controllers read RFID tag issued to the user and search 
this tag number in non-volatile RAM. On a successful match, the controllers request the computer terminal to capture the user image. The computer system uses neural network trained face recognition module to verify the user authenticity and responds to the controllers by sending them 'access granted' or 'access denied' message. The controllers grant the access to the user or make emergency call accordingly. This system is made centralized with the help of a web server. The web server takes information from computer terminals in hostels and keeps track of a particular user. Although the developed system is useful in reducing security threats to the hostels, there is a room for improvement in the response time of the system. The response time can be improved by using dedicated processors instead of computer systems capable of processing the images in real time.

\section{REFERENCES}

[1] D. L. Wu, Wing W. Y. NG, D. S. Yeung,... and..H...L...Ding,..."A brief survey on current RFID applications," in Proc. International Conference on Machine Learning and Cybernatics, Baoding, July 12-15, 2009, pp. 2330-2334.

[2] B. Yan and D. Y. Lee, "Design of spot ticket management system based on RFID," in Proc. International Conference on Networks Security, Wireless Communications and Trusted Computing, 2009, pp 496-499.

[3] G. Ostojic, S. Stankovski, and M. Lazarevic, "Implementation of RFID technology in parking lot access control system," in Proc. Annual RFID Eurasia Conference, 2007, pp. 1-5.

[4] N. Ahmad, S. Butler, and U. Ramachandran, "GuardianAngel: An RFID based indoor guidance and monitoring system," 2010, pp. 546-551.

[5] K. S. Huang and S. M. Tang, "RFID applications strategy and deployment in bike renting system," in Proc. ICACT 2008, pp. 660-663.

[6] S. Lahiri, RFID sourcebook, IBM Press, Westford, Massachusetts, 2006.

[7] F. Lourenco and C. Almeida, "RFID based monitoring and access control system," in Proc. INFORUM, 2009.

[8] R. Weinstein, "RFID: A technical overview and its application to the enterprise," IT Professional, vol. 7, no. 3, May-June 2005, pp. 27-33.

[9] X. L. Meng, Z. W. Song, and X. Y. Li, "RFID-Based security authentication system based on a novel face-recognition structure," in Proc. WASE International Conference on Information Engineering, 2010, pp. 97-100.

[10] D. L. Wu, Wing W. Y. NG, Patrick P. K. Chan, H. L. Ding, B. Z. Jing, and D. S. Yeung, "Access control by RFID and face recognition based on neural network," in Proc. International Conference on Machine Learning and Cybernatics, July 11-14, 2010, pp. 675-680.

[11] M. A. Mazidi, J. C. Mazidi, and R. D. Mckinaly, The 8051 Microcontroller and Embedded Systems, Pearson Education, 2006.

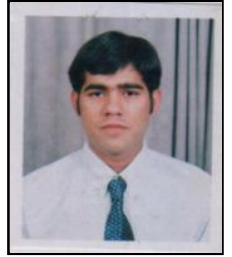

Umar Farooq received his B.Sc. and M.Sc. both in electrical engineering from University of Engineering \& Technology Lahore in 2004 and 2010 respectively. $\mathrm{He}$ is currently with the Department of Electrical Engineering, University of The Punjab Lahore. His research interests include the application of intelligent techniques to problems in control engineering, robotics and power electronics.

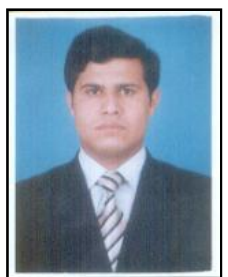

Mahmood ul Hasan received his B.Sc. in electrical engineering from University of Engineering \& Technology Lahore in 2006. He is the recipient of prestigious silver and bronze medal awards in his pre engineering and secondary school studies respectively. $\mathrm{He}$ is with Department of Electrical Engineering Mirpur University of Science and Technology Azad Kashmir and is working towards M.Sc. degree in electrical engineering from University of Engineering \& Technology Lahore. His research interests include electronic instrumentation, intelligent control and robotics.

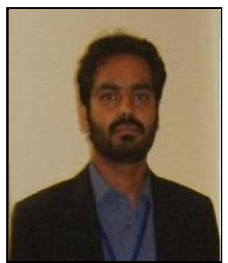

Muhammad Amar received his B.Sc. in electrical engineering from University of The Punjab Lahore in 2010 and M.Sc. in Electrical Engineering from University of Engineering \& Technology Lahore in 2012. He is currently working towards Ph.D. degree in electrical engineering from Monash University, Australia. His research interests include the application of intelligent techniques to problems in control engineering, robotics and machine vision.

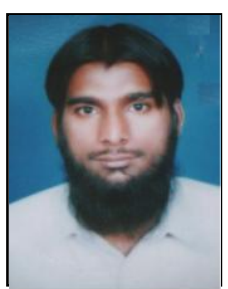

Athar Hanif holds B.Sc. and M.Sc. degrees in electrical engineering from University of Engineering \& Technology Taxila and University of Engineering \& Technology Lahore respectively. He is currently working towards the Ph.D. degree in control engineering from Muhammad Ali Jinnah University Islamabad. $\mathrm{He}$ is with Department of Electrical Engineering, The University of Lahore where he is working as Assistant Professor. His research interests include the robust nonlinear control of hybrid vehicles and power converters.

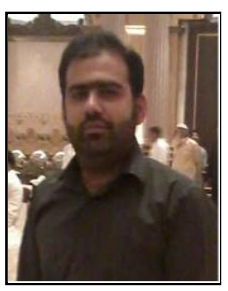

Muhammad Usman Asad received his B.Sc. in Electrical Engineering from University of The Punjab Lahore in 2010. During his stay at electrical engineering Department University of The Punjab Lahore, he served as President of Society of Engineering Excellence (2009) and contributed in the research activities of the society. He is the recipient of Gold Medal award for his paper on Ball Scoring Robot in $24^{\text {th }}$ IEEEP International Multi-topic Symposium, 2009 and Silver Medal award for his paper on Neural Controller for Robot Navigation in $26^{\text {th }}$ IEEEP International Multi-topic Symposium, 2011. He is currently working towards M.Sc. degree in electrical engineering from G.C. University Lahore. He is with Department of Electrical Engineering, The University of Lahore where he is a Lecturer. His research interests include intelligent control of Robotics and Power systems. 